\title{
Propuesta de un programa educativo para padres de niños con cardiopatías congénitas
}

\author{
Educational program for congenital cardiopathy \\ children's parents
}

\author{
Enf. Paola Pino Armijo ${ }^{a}$, Enf. Carmen Gloria Valdés Valenzuela ${ }^{b}$ \\ Enf. Paula Fajuri Moyano ${ }^{b}$, Enf. Olga Garrido Villablanca ${ }^{a}$ y Dr. Andrés Castillo Moya ${ }^{b}$
}

\section{RESUMEN}

Los niños con cardiopatías congénitas se ven enfrentados a una serie de procedimientos en forma cronológica y secuenciada. Durante todo este proceso, tanto el niño como sus padres requieren información oportuna, educación y preparación para el alta. No obstante, la información acerca de cada uno de los aspectos afectados por la enfermedad generalmente es abordada en forma aislada por los distintos profesionales y no como parte de un programa educativo integral.

El programa educativo debe considerar la naturaleza de la enfermedad, de los usuarios y de los educadores durante su planificación, y debe ser implementado por un equipo multidisciplinario, en forma continua durante la hospitalización y el seguimiento, utilizando los distintos métodos educativos disponibles e incorporando los contenidos mínimos descritos en la literatura.

Es objetivo de esta revisión identificar las dimensiones y elementos claves para considerar en el diseño de un programa educativo para padres de niños con cardiopatías congénitas basado en el modelo de Kaufman.

Palabras clave: programa educativo, educación, padres, cardiopatías congénitas.

\section{ABSTRACT}

The children with congenital heart disease are faced with a series of procedures in chronological sequence. Throughout this process the infant and their parents require timely information, education and preparation for discharge. However, the information about each of the aspects affected by the disease is usually addressed in isolation by different professionals and not as part of a comprehensive educational program.

The educational program should consider the nature of the disease, of the users and educators during their planning and must be implemented by a multidisciplinary team, continuously during hospitalization and follow-up, using various teaching methods available, and incorporating the minimum content described in the literature. The objective of this review is to identify the dimensions and key elements to consider in the design of an educational program for parents of children with congenital heart disease based on the model of Kaufman.

Keywords: educational program, education, parents, congenital heart defects. http:/ /dx.doi.org/10.5546/aap.2014.451

\section{INTRODUCCIÓN}

Las cardiopatías congénitas (CC) son las malformaciones congénitas más frecuentes y constituyen un problema relevante en salud a nivel mundial. ${ }^{1}$

Los niños con CC se ven enfrentados a una serie de procedimientos en forma cronológica y secuenciada; entre ellos, control médico, exámenes a mbulatorios, confirmación diagnóstica, tratamiento médico, traslado a un centro de referencia, tratamiento quirúrgico, traslado a su hospital de base, alta y seguimiento posterior. Durante todo este proceso, tanto el niño como sus padres pueden presentar estrés, angustia e incertidumbre, por lo que requieren información oportuna, educación permanente y preparación para el alta. ${ }^{2}$ Sin embargo, la educación en torno a la cardiopatía, al tratamiento médico y a la cirugía cardíaca es realizada generalmente en forma incidental o poco formal.

Por este motivo, la Asociación de Afectados por Cardiopatías Congénitas de Cataluña sugiere que los profesionales de la salud deben disponer de información detallada acerca de la evolución de la enfermedad, las secuelas o complicaciones de las intervenciones quirúrgicas, el ingreso a la UCI, la edad del niño al momento de cada intervención, la posición familiar respecto de la CC y las posibilidades del paciente y su familia de usar toda la información y recursos disponibles para afrontar la enfermedad. ${ }^{3}$ No 
obstante, la información acerca de cada uno de los aspectos afectados por la enfermedad generalmente es abordada en forma aislada por los distintos profesionales y no como parte de un programa educativo integral.

Según Kaufman, el enfoque sistemático es un proceso que se aplica para identificar y resolver importantes problemas educativos, el cual es indispensable para el diseño de programas educativos humanos y pertinentes. ${ }^{4}$

Es objetivo de esta revisión identificar las dimensiones y los elementos clave para considerar en el diseño de un programa educativo para padres de niños con CC basado en el modelo de Kaufman.

\section{DIMENSIONES PARA CONSIDERAR EN EL DISEÑO DEL PROGRAMA EDUCATIVO}

Según el modelo de Kaufman, los profesionales deben considerar tres dimensiones durante la planificación de un programa educativo: naturaleza de la enfermedad, naturaleza de los usuarios y naturaleza de los educadores. ${ }^{4}$

\section{Naturaleza de la enfermedad}

Se denominan CC a todas las malformaciones cardíacas que están presentes al momento del nacimiento. Su incidencia es de entre 4 y 12 por 1000 recién nacidos vivos, independiente de factores, como raza, condición socioeconómica o situación geográfica. ${ }^{1}$

En Chile, las anomalías congénitas constituyen la segunda causa de muerte en los menores de un año y son responsables de un $32 \%$ de las defunciones en este grupo de edad. La mortalidad por CC en niños menores de 1 año supone alrededor de 1/10 de todas las muertes en ese período de la vida. ${ }^{1}$

Existen alrededor de 150 CC diagnosticables, y cada una de ellas puede tener diferentes grados de gravedad. No todos los afectados responden igual, aunque padezcan el mismo tipo de cardiopatía. Esto hace que el equipo de salud se enfrente con pacientes que comparten características comunes, pero con repercusiones diferentes, y se destaca que la mayoría de ellos pasa algún tiempo hospitalizados, incluso desde el nacimiento. ${ }^{3}$

En el Hospital Clínico UC, durante la última década, fueron realizadas 1674 cirugías cardíacas en 1344 pacientes con CC menores de 18 años (1,25 cirugías por paciente). La mortalidad operatoria en este período fue $5,9 \%{ }^{5}$

\section{Naturaleza de los usuarios}

La complejidad y la cronicidad atribuida a las CC afecta directamente la salud y la calidad de vida de los padres, quienes presentan altos niveles de estrés, angustia e incertidumbre durante la hospitalización, además de un aumento en la demanda y carga de cuidado en el hogar. 2,6-13

Estrés, angustia e incertidumbre durante la hospitalización: La experiencia se evidencia para los padres como un proceso que implica sufrimiento; además, la falta de cuidado percibido, la escasa atención y protección otorgada por el equipo de salud acentúan emociones, como miedo, temor, soledad, rabia e impotencia por su situación de vida. ${ }^{6}$

En el momento del diagnóstico, los padres sienten que "se les viene el mundo entero encima". En la primera etapa, sienten incredulidad, culpa y soledad. Luego sienten desconfianza acerca del diagnóstico y tratamiento, por lo que buscan garantías y seguridad. Cabe destacar que las funciones emocionales que se le atribuyen al corazón añaden una importante carga sentimental a este problema. ${ }^{7}$

Durante la cirugía, se evidencia un momento de crisis familiar, manifestado por una mezcla de sentimientos, como miedo a la muerte, a la cirugía y a la anestesia, y culpa e impotencia frente a las diferentes etapas del tratamiento; sin embargo, también representa la posibilidad de curación y aumento de la calidad de vida de su hijo. ${ }^{8}$ Los padres perciben altos niveles de estrés durante la hospitalización de sus hijos, con diferencias significativas en relación con el nivel socioeconómico y con el lugar de residencia de los padres, pero sin diferencias en relación con la gravedad de la enfermedad, con los días de hospitalización ni con el sexo de los padres. ${ }^{2}$ Sin embargo, en otro estudio, el nivel de estrés se relacionó significativamente con la edad de los niños, con el apoyo social y con el nivel de incertidumbre de los padres, provocado por la ambigüedad en los criterios para el cuidado de su hijo, la falta de claridad y de información acerca del tratamiento y seguimiento. ${ }^{9}$

Durante la hospitalización, las madres sienten angustia y un gran impacto visual, pero luego motivación y esperanza al descubrir la capacidad de recuperación de sus hijos. ${ }^{7}$ No se consideran solo acompañantes del niño, sino hospitalizadas junto con ellos, comparten sus sufrimientos e incluso renuncian a su propia vida, a sus sentimientos y a sus necesidades, y se brindan por completo en el proceso de recuperación de su hijo, 
tratando de satisfacer sus necesidades, como una extensión de los cuidados de enfermería. ${ }^{10}$

$\mathrm{Al}$ abandonar la UCI, nuevamente sienten inseguridad, temor a contagios y déficit en la atención recibida. Finalmente, durante la preparación para el alta, sienten felicidad, pero también terror al alejarse del amparo del hospital, por lo que buscan toda la información posible acerca de los cuidados que debe recibir su hijo en el hogar. ${ }^{7}$

Demanda y carga de cuidado en el hogar: Los padres perciben alterada su calidad de vida. En un estudio, los padres perciben más afectada la dimensión psicológica (ansiedad, preocupación por el futuro y las complicaciones, temor a nuevos embarazos, trastornos del sueño, cambios en la relación de pareja, sentimientos de tristeza y abandono), seguida de la dimensión funcional (escaso apoyo en la distribución de las actividades del cuidado), la dimensión social (cambios en la actividad laboral y preocupaciones económicas) y la gravedad de la enfermedad (temor a las descompensaciones, cirugía, discriminación, maltrato y muerte). ${ }^{11}$ Sin embargo, en otro estudio, los padres perciben más afectado el bienestar espiritual (incertidumbre generada por la enfermedad), seguido por el bienestar social (aislamiento social), el bienestar físico (trastornos del sueño, fatiga y dolor) y el bienestar psicológico (miedo a la recaída, avance a una segunda enfermedad y angustia por el diagnóstico y tratamiento). ${ }^{12}$ Esta percepción se relacionó significativamente con la edad y el nivel socioeconómico de los padres, con el tiempo de cuidado y con ser el único cuidador. ${ }^{13}$

\section{Naturaleza de los educadores}

Un cuestionario aplicado a 389 padres de niños con cardiopatías congénitas durante una consulta ambulatoria reveló que la principal fuente de información médica para este grupo fue a través del cardiólogo infantil (83\%), seguido por el médico familiar (53\%), el pediatra general (40\%) y finalmente a través de internet $(15 \%){ }^{14}$
La educación recibida por los padres a través de las distintas fuentes de información se puede evaluar a través del nivel de conocimiento de los usuarios. En la Tabla 1, se describen los resultados reportados por estudios internacionales respecto a los conocimientos de los padres de niños con CC acerca de la enfermedad cardíaca. ${ }^{15-17}$ El nivel de conocimiento se relacionó significativamente con el tipo de CC, con la ocupación y con el nivel educacional de los padres. ${ }^{16}$

Los padres presentan un nivel de conocimiento adecuado respecto al nombre y naturaleza de la malformación cardíaca. Los autores refieren que este tema es abordado tanto al momento del diagnóstico como durante la hospitalización y controles posteriores, y que los profesionales hacen un esfuerzo por entregar esta información en forma clara y utilizando diagramas de color con el fin de facilitar la compresión. . $^{15,16}$

Sin embargo, presentan menor conocimiento en relación con el nombre y función de los medicamentos, efectos secundarios e interacciones. Los autores sugieren que el uso de lenguaje técnico puede ser una dificultad importante, pero que también existe la percepción de que los padres no necesitan una comprensión profunda respecto al tema ya que tienen la posibilidad de contactarse con un cardiólogo en caso de ser necesario. ${ }^{15,16}$

El menor nivel de conocimiento se presenta en la prevención de endocarditis bacteriana y el nivel adecuado de actividad física. Los autores proponen que la comprensión de ambos temas podría mejorarse a través de información verbal más completa antes del alta y en las consultas posteriores, y a través de folletos educativos específicos. ${ }^{15-17}$

Además, el nivel de conocimiento se relaciona significativamente con las necesidades educativas de los usuarios, y existe una relación negativa entre ambas variables. ${ }^{18}$ En la Tabla 2, se describen los resultados reportados a nivel mundial en relación con las necesidades educativas de los padres de niños con CC acerca de la enfermedad cardíaca. ${ }^{18-20}$

TABLA 1. Conocimientos de los padres de niños con cardiopatías congénitas acerca de la enfermedad cardíaca

\begin{tabular}{lcccc}
\hline Estudios & $\begin{array}{c}\text { Nombre } y \\
\text { naturaleza de la CC }\end{array}$ & $\begin{array}{c}\text { Nombre y función } \\
\text { de los medicamentos }\end{array}$ & $\begin{array}{c}\text { Prevención de } \\
\text { endocarditis bacteriana }\end{array}$ & $\begin{array}{c}\text { Nivel adecuado de } \\
\text { actividad física }\end{array}$ \\
\hline Chessa, et al. $(\mathrm{n}=148)$ & $91,2 \%$ & $34,7 \%$ & $24,3 \%$ & $41,2 \%$ \\
Cheuk, et al. $(\mathrm{n}=156)$ & $59 \%$ & $44,6 \%$ & $26,9 \%$ & $59 \%$ \\
Hagg, et al. $(\mathrm{n}=90)$ & $38,9 \%$ & $87,8 \%$ & $16,7 \%$ & - \\
\hline
\end{tabular}

CC: cardiopatías congénitas. 
Los autores concuerdan en que existe una diferencia entre el nivel de conocimientos y el interés por conocer más acerca de los tres temas estudiados, que es estadísticamente significativa en el caso de la prevención preconcepcional de CC. ${ }^{18-20}$

Además, proponen que es necesario abordar estos temas precozmente y profundizar en ellos durante los controles posteriores. ${ }^{19,20}$ En este sentido, uno de los estudios demostró que el seguimiento telefónico realizado por un fisioterapeuta especialista en cardiología es un método simple y fácil de implementar, cuya duración promedio fue de 10 minutos por paciente, y que permite identificar problemas en el nivel de actividad física y sugerir cambios en los programas de ejercicio cuando estos impliquen un riesgo. ${ }^{19}$

Estos hallazgos develan que el nivel de conocimientos de los padres de niños con CC respecto a la enfermedad de su hijo es deficiente y que la educación otorgada en la actualidad debe ser revisada ya que no sería adecuada para promover la comprensión de los padres acerca de la enfermedad ni para mejorar sus prácticas de cuidado en el hogar. ${ }^{15-20}$ Cabe destacar que un programa educativo formal implementado en España para 60 pacientes trasplantados de corazón aumentó significativamente los conocimientos con respecto al rechazo, las infecciones y los signos y síntomas de alerta, y mejoró las actitudes y prácticas de autocuidado en relación con el tratamiento inmunosupresor, lo que contribuyó a la adquisición de habilidades que permiten que los usuarios sean más autónomos y menos dependientes del sistema de salud. ${ }^{21}$

\section{ELEMENTOS CLAVE PARA CONSIDERAR EN EL DISEÑO DEL PROGRAMA EDUCATIVO}

Según el modelo de Kaufman, los profesionales deben considerar cinco elementos clave durante la implementación de un programa educativo: educador, momento, lugar, medios y contenidos. $^{4}$

\section{Educador}

El programa educativo debe ser implementado por un equipo multidisciplinario..$^{3,21-26} \mathrm{El}$ médico tratante proporciona la información inicial y el apoyo a la familia durante el diagnóstico, referencia y seguimiento posterior. El cardiólogo y el cardiocirujano proporcionan la base para la comprensión del diagnóstico, pronóstico, tratamiento médico y quirúrgico. Las enfermeras facilitan la comprensión acerca de la fisiopatología, intervenciones, recuperación y prevención de complicaciones..$^{22}$ Las nutricionistas ofrecen alternativas para complementar los nutrientes según los requerimientos del paciente. Los kinesiólogos ofrecen alternativas para incrementar progresivamente la actividad física y reincorporar al niño a sus actividades. ${ }^{23} \mathrm{La}$ psicopedagoga proporciona conocimientos a través del juego y ofrece un sentido de normalidad con actividades apropiadas para el desarrollo del niño. El psiquiatra y el psicólogo infantil intervienen en caso de depresión, ansiedad y hospitalización prolongada. ${ }^{22}$

Cabe destacar que la enfermera es el puente entre los padres y el resto del equipo de salud. Es deber del equipo de enfermería otorgar el tiempo necesario a los padres para aceptar la enfermedad de su hijo; escuchar y aconsejar, manteniendo una actitud comprensiva; explicar por qué y para qué se realizan exámenes y tratamientos; enseñar cómo realizar los cuidados del niño para incluir a los padres en él; estar bien informado de la evolución del niño para evitar incongruencias; y trasmitir la información de manera positiva, pero sin crear falsas expectativas. ${ }^{27}$

\section{Momento y lugar}

El programa educativo debe ser implementado en forma continua durante todas las etapas de la hospitalización y el seguimiento. Al momento del ingreso, los pacientes y sus padres deben ser informados acerca de la preparación para la cirugía cardíaca. ${ }^{22}$ Durante la hospitalización, los pacientes y sus padres deben recibir educación en forma continua, una vez que el paciente

TABLA 2. Necesidades educativas de los padres de niños con cardiopatías congénitas acerca de la enfermedad cardíaca

\begin{tabular}{lccc}
\hline Estudios & Tema & $\begin{array}{c}\text { Conocimiento } \\
\text { acerca del tema }\end{array}$ & $\begin{array}{c}\text { Interés en conocer } \\
\text { más acerca del tema }\end{array}$ \\
\hline Blanco, et al. $(\mathrm{n}=474)$ & Prevención preconcepcional de CC & $17,8 \%$ & $65,6 \%$ \\
Kendall, et al. $(\mathrm{n}=253)$ & Nivel adecuado de actividad física & $35 \%$ & $54 \%$ \\
Fernandes, et al. $(\mathrm{n}=500)$ & Cuidados a largo plazo & $44 \%$ & $96 \%$ \\
\hline
\end{tabular}

CC: cardiopatías congénitas. 
se encuentre estable. ${ }^{21} \mathrm{Al}$ momento del alta, es fundamental continuar con un programa complementario de manera externa ya que, durante esta etapa, el niño y sus padres deben reforzar lo aprendido en el hospital y, además, deben lograr la mejoría en aspectos, como condición física del niño y control de los factores de riesgo cardiovascular..$^{24,25}$

\section{Medios}

El programa educativo debe ser implementado considerando los distintos métodos educativos disponibles. ${ }^{21,22,26}$ Las sesiones verbales deben realizarse utilizando un lenguaje simple, concreto con la sensibilidad del niño y evitando palabras que puedan ser interpretadas como amenaza. Los folletos educativos deben realizarse utilizando un lenguaje claro y preciso, con imágenes visuales o diagramas. Los talleres grupales deben realizarse formando grupos pequeños de trabajo, en sesiones cortas de 30 minutos. Los métodos indirectos deben realizarse a través de videos, diapositivas, sesiones estructuradas de juego, recorridos por el hospital e internet. ${ }^{22}$

Cabe destacar que una gran cantidad de padres está utilizando el internet para educarse sobre la cardiopatía de su hijo, y la mayoría de ellos considera que la información obtenida facilita la comprensión de la enfermedad. Es deber del equipo de salud supervisar el uso de este método, identificando previamente las referencias apropiadas para los padres, con el fin de evitar que estos accedan a información errónea. ${ }^{14}$

\section{Contenidos}

El programa educativo debe ser implementado considerando los contenidos mínimos descritos en la literatura:

Aspectos generales de la cardiopatía: Características de la enfermedad (evolución y pronóstico), tratamiento médico (función, dosis, reacciones adversas, interacciones con otros medicamentos) y tratamiento quirúrgico (tipo y número de cirugías que requiere el niño). $3,7,8,26,28,29$

Características de la cirugía cardíaca: Preparación preoperatoria, tipo de anestesia, posibles secuelas o complicaciones posteriores, necesidad de hospitalización en UCI y apariencia del niño después de la cirugía. $3,7,8,22,26$

Cuidados posteriores a la cirugía cardíaca: Rutina del hospital, duración de la intubación y de la monitorización, presencia de dispositivos invasivos (catéteres, sondas, drenajes, cables de marcapaso) y cuidados de la piel y de la herida operatoria. $7,8,22,26$

Participación de los padres en el cuidado: Posibilidad de acompañar a su hijo y de participar en las rutinas de cuidado durante la hospitalización (alimentación, higiene, recreación), impacto que genera la experiencia en el comportamiento del niño, qué deben esperar durante la hospitalización, qué funciones deben desempeñar y cómo pueden apoyar a su hijo. ${ }^{78,22,29}$

Reincorporación a las actividades de la vida diaria: Alternativas para incrementar progresivamente la actividad física del niño (muchas veces restringida por indicación médica, sobreprotección familiar e inseguridad del niño). Es importante considerar la intensidad del ejercicio, la duración, el riesgo de colisión, la necesidad de entrenamiento y el estrés que este puede causar. ${ }^{7,23-25,28,30}$

Prevención y detección de complicaciones del desarrollo: Factores de riesgo (edad de la cirugía, hospitalización prolongada), medidas de prevención (reconocer la importancia del contacto físico, ofrecer un plan de estimulación psicomotriz con metas específicas, evitar la sobreprotección) y signos y síntomas de alerta. ${ }^{26,28,30,31}$

Prevención y detección de complicaciones nutricionales: Factores de riesgo (CC cianóticas, insuficiencia cardíaca), medidas de prevención (favorecer la lactancia materna, alimentar en forma fraccionada, ofrecer alternativas para complementar los nutrientes, considerar la alimentación enteral) y signos y síntomas de alerta. ${ }^{26,28,30}$

Prevención y detección de complicaciones respiratorias: Factores de riesgo (prematurez, desnutrición, cromosomopatías, enfermedades respiratorias crónicas, tener hermanos menores de 11 años), medidas de prevención (realizar lavado de manos, evitar lugares con muchos niños y el tabaquismo en el hogar, reconocer la importancia de la vacunación para neumococo, influenza, varicela y VRS) y signos y síntomas de alerta. ${ }^{28,30,32}$

Prevención y detección de complicaciones cardiovasculares: Factores de riesgo, medidas de prevención (controlar el peso, realizar actividad física, tener una dieta cardiosaludable, manejar las emociones y el estrés, evitar el consumo de tabaco y la inhalación pasiva de humo) y signos y síntomas de alerta. ${ }^{24,25,30}$

\section{CONCLUSIÓN}

Es esencial que las unidades que atienden a niños con CC diseñen programas educativos humanos y pertinentes, que promuevan la comprensión de los padres acerca de la 
enfermedad y que mejoren sus prácticas de cuidado en el hogar para optimizar así la calidad del cuidado de enfermería.

Es así como el programa educativo para padres de niños con CC debe considerar las características identificadas respecto a la naturaleza de la enfermedad, de los usuarios y de los educadores durante su planificación. Además, este programa debe ser implementado por un equipo multidisciplinario, en forma continua durante todas las etapas de la hospitalización y el seguimiento, utilizando los distintos métodos educativos disponibles e incorporando los contenidos mínimos descritos en la literatura en relación con los aspectos generales de la cardiopatía, las características de la cirugía cardíaca, los cuidados posteriores a la cirugía y los cuidados al momento del alta.

\section{REFERENCIAS}

1. Chile. Ministerio de Salud. Guía Clínica: Cardiopatías Congénitas Operables en menores de 15 años. [Internet]. Santiago: Ministerio de Salud;2010. Disponible en: http:/ / web.minsal.cl/portal/url/item/720bfefe91e0d2ede04001 011f010ff2.pdf. [Consulta: 7 de mayo de 2014].

2. Franck LS, McQuillan A, Wray J, Grocott MP, et al. Parent stress levels during children's hospital recovery after congenital heart surgery. Pediatr Cardiol 2010;31(7):961-8.

3. Scott-Tennent Y. Servicio de asesoramiento a los profesionales de la enseñanza que atienden a niños y jóvenes afectados de cardiopatía congénita. Univ Tarracon Rev Ciènc Educ 2007;XXXI:209-21.

4. Kaufman R. Planificación de sistemas educativos: ideas básicas concretas. México DF: Trillas, 1973.

5. Clavería C, Cerda J, Becker P, Schiele C, et al. Mortalidad operatoria y estratificación de riesgo en pacientes pediátricos operados de cardiopatía congénita: experiencia de 10 años. Rev Chil Cardiol 2014;33(1):11-9.

6. Gallardo A. Experiencia de una madre con hijo con malformación. Horiz Enferm 2011;22(1):45-54.

7. Da Rocha Guisande R. La visión de los padres. Bol Pediatr 2003;43(184):125-8.

8. Salgado CL, Lamy ZC, Nina RV, de Melo LA, etal.Pediatric cardiac surgery under the parents sight: a qualitative study. Rev Bras Cir Cardiovasc 2011;26(1):36-42.

9. Sunhee L, Ji-Soo Y, Il-Young Y. Parenting stress in mothers of children with congenital heart disease. Asian Nurs Res 2007;1(2):116-24.

10. Rocha $\mathrm{D}$, Zagonel I. Modelo de cuidado transicional à mãe da criança com cardiopatia congênita. Acta Paul Enferm 2009;22(3):243-9.

11. De León Ojeda NE. Calidad de vida y perspectiva del cuidador en niños con defectos cardiovasculares congénitos. Bioética 2011;11(1):10-22.

12. Montalvo Prieto A, Romero Massa E, Flórez Torres IE. Percepción de la calidad de vida de cuidadores de niños con cardiopatía congénita Cartagena, Colombia. Invest Educ Enferm 2011;29(1):9-18.

13. Romero Massa E, Montalvo Prieto A, Flórez Torres I. Características de los cuidadores de niños con cardiopatías congénitas complejas y su calidad de vida. Av Enferm 2010;28(1):39-50.

14. Massin MM, Montesanti J, Gerard P. Use of the Internet by parents of children with congenital heart disease. Acta Cardiol 2006;61(4):406-10.

15. Chessa M, De Rosa G, Pardeo M, Negura DG, et al. What do parents know about the malformations afflicting the hearts of their children? Cardiol Young 2005;15(2):125-9.

16. Cheuk DK, Wong SM, Choi YP, Chau AK, Cheung YF. Parents' understanding of their child's congenital heart disease. Heart 2004;90(4):435-9.

17. Haag F, Casonato S, Varela F, Firpo C. Parents' knowledge of infective endocarditis in children with congenital heart disease. Rev Bras Cir Cardiovasc 2011;26(3):413-8.

18. Blanco Pereira ME, Almeida CamposS, Olivera Muñoz EH, Fernández Arenas C, etal. Necesidades de aprendizaje sobre prevención preconcepcional de cardiopatías congénitas en el Policlínico “Carlos Verdugo". Rev Méd Electrón. 2009;31(3). Disponible en: http://scielo.sld.cu/pdf/rme/v31n3/ spu02309.pdf. [Consulta: 17 de noviembre de 2011].

19. Kendall L, Parsons JM, Sloper P, Lewin RJ. A simple screening method for determining knowledge of the appropriate levels of activity and risk behaviour in young people with congenital cardiac conditions. Cardiol Young 2007;17(2):151-7.

20. Fernandes SM, Verstappen A, Ackerman K, Adams EE, et al. Parental knowledge regarding lifelong congenital cardiac care. Pediatrics 2011;128(6):e1489-95.

21. Solís Muñoz M, García López F, Casado Dones MJ, Iza Zabala A, et al. Programa de educación para la salud en el trasplantado de corazón. Ensayo controlado con asignación aleatoria. Enferm Clin 2005;15(3):131-40.

22. LeRoy S, Elixson EM, O`Brien P, Tong E, et al. Recommendations for preparing children and adolescents for invasive cardiac procedures: a statement from the American Heart Association Pediatric Nursing Subcommittee of the Council on Cardiovascular Nursing in collaboration with the Council on Cardiovascular Diseases of the Young. Circulation 2003;108(20):2550-64.

23. Silva ME, Feuser MR, Silva MP, Uhlig S, et al. Pediatric cardiac surgery: what to expect from physiotherapeutic intervention? Rev Bras Cir Cardiovasc 2011;26(2):264-72.

24. Ilarraza Lomeli H, Quiroga P, Rius Suárez MD. Rehabilitación cardíaca en población pediátrica. Más allá que ayudar a un niño a readaptar su corazón. Arch Cardiol Mex 2008;78(2):129-33.

25. Marugan Torres P. Cardiopatía congénita. En: Portuondo Maseda MT, Martínez Castellanos T, Delgado Pacheco J, García Hernández P, et al, eds. Manual de Enfermería en Prevención y Rehabilitación Cardiaca. Madrid: Asociación Española de Enfermería en Cardiología; 2009. Págs.229-32.

26. Pye S, Green A. Parent education after newborn congenital heart surgery. Adv Neonatal Care 2003;3(3):147-56.

27. Rodríguez M. El mundo de las cardiopatías congénitas. Enferm Cardiol 2003;X(28):16-7.

28. Torre Rodríguez I. Problemas del niño con cardiopatía. La visión del pediatra. Bol Pediatr 2003;43(184):122-4.

29. Alonso Fernández S, Ros Elvira MT. Plan de cuidados al niño con una cardiopatía congénita: Utilizando NANDA, NIC y NOC. Enferm Cardiol 2008;XV(44):33-6.

30. Picazo Angelin B. Inmunizaciones y seguimiento pediátrico del lactante con cardiopatía congénita. En:Zabala Argüelles JI, ed. Protocolos Diagnósticos y Terapéuticos en Cardiología Pediátrica. Madrid: Asociación Española de Pediatría; 2005. Págs.1-9.

31. Díaz Benítez ER. Lactante menor con alteración de las necesidades básicas secundario a cardiopatía congénita. Rev Mex Enf Cardiol 2011;19(2):74-9.

32. Medrano López C, García-Guereta Silva L. Infecciones respiratorias y cardiopatías congénitas: dos estaciones del estudio CIVIC. An Pediatr 2007;67(4):329-36. 